\title{
Cloud and rain effects on ALTIKA/SARAL Ka band radar altimeter. Part II: Definition of a rain/cloud flag
}

\author{
Jean Tournadre, Juliette Lambin and Nathalie Steunou
}

\begin{abstract}
The main instrument of the French-Indian Altika/SARAL mission scheduled for launch in 2010 , is the Kaband Altika altimeter. The high attenuation due to atmospheric water (liquid or vapor) at this frequency band is the major drawback of the use of Ka-band. In part I of this paper, the impact of rain/clouds on Ka-band data and on the accuracy of the estimates of the geophysical parameters have been analyzed and quantified using an analytical model of waveform. Waveform distortion and errors on the geophysical parameters can be significant especially for small dense clouds and rain cells. It is thus necessary to flag the data potentially affected by rain and clouds. The use of a single channel for Altika prevents the use of the classical dual-frequency rain flag used for Topex or Jason altimeters, and requires the definition of a new flag based on the altimeter signal alone. Past studies showed that clouds and rain are characterized by sharp coherent along-track fluctuations of the off-nadir angle estimates. The new flagging algorithm is based on the analysis of the variations of this parameter by Matching Pursuit (MP) algorithm. MP allows the decomposition of a signal into a few salient features or atoms chosen from a dictionary of elementary functions. The dictionary is here defined by the wavelet decomposition of the signal. The method has been tested on an ensemble of Altika passes simulated for cloudy, rainy and cloud/rain free situations. The false alarm rate is almost nil while the detection performances are better than $90 \%$ at a range error of $5 \mathrm{~cm}$ and significant wave height error of $20 \mathrm{~cm}$. The flag can be easily adapted to other altimeters' data and has been used to flag several Jason-1 passes. The comparison to the operational dual-frequency flag shows that the MP flag performs better in detecting range errors and waveforms distortion, while its performances are inferior in detecting samples attenuated by rain.
\end{abstract}

Index Terms-Ka-band altimeter, rain and cloud impact, rain/cloud flag.

\section{INTRODUCTION}

$\mathbf{T}$ HE main instrument of the AltiKa/SARAL mission, developed by the French Centre National d'Etudes Spatiales (CNES) in cooperation with Indian Space Research Organization (ISRO ), is based on the wide-band Ka-band Altika altimeter. The major drawback of the use of Ka-band is that the attenuation of the signal due to liquid water (rain and clouds, which are often dense and frequent in the Tropics), is high. This will be a strong constraining factor, as limitation of the altimeter link budget imposes an attenuation of the signal of less than $3 \mathrm{~dB}$. In part I of the paper [1] an analytical model,

J. Tournadre is with IFREMER (Institut Français de Recherche pour l'Exploitation de la Mer), Laboratoire d'Océographie Physique et Spatiale, IFREMER, 29280 Plouzané, France, e-mail: jean.tournadre@ifremer.fr

J. Lambin and N. Steunou are with Centre National D'Etudes Spatiales, 31401 Toulouse cedex9 FRANCE. based on the one developed by [2] for Ku-band altimeters, has been adapted to compute Ka-band altimeter waveforms in presence of cloud and rain. It has been used to model and quantify the impact of rain and cloud on Altika waveforms as well as on the accuracy of the geophysical parameters estimates. The results showed that even light rain and/or cloud liquid water content, especially for small rain cells and/or clouds, can significantly distort the echo waveform and degrade the accuracy of the geophysical parameters inferred by waveform analysis. It is thus necessary to detect and flag the samples potentially affected by atmospheric liquid water. The same problem was encountered for Ku-band altimeters, such as Topex, Jason-1 or Envisat, for medium and heavy rain. For these dual frequency altimeters rain flags, based on the differential attenuation of the main (Ku-band) and secondary channels (C-band for Topex and Jason-1 and S-band for Envisat) by rain droplets, were defined and are currently used operationally [3], [4], [5], [6]. Unfortunately, Altika will be a single frequency altimeter and this kind of simple and efficient flag can obviously not be utilized.

Rain flags based on simple threshold on atmospheric liquid water content estimates from coincident microwave radiometer data, as originally used for Topex, were shown by [3] and [6] to have a high false alarm rate for high liquid water content and a low performance for low liquid water content. Furthermore, the distortion of waveforms by rain or clouds depends more on the variability of liquid water within the altimeter footprint than on the average content itself [1]. This, and the high sensitivity of Ka-band to liquid water, imply that this kind of rain/cloud flag can certainly not perform efficiently. It is thus necessary to develop a new rain flag and it has to be based on the analysis of the altimeter measurement alone.

Past experience with Ku-band altimeter data showed that rain cells are characterized by sharp coherent along-track variations of measured backscatter and off-nadir angle estimates [2]. Altika waveforms modeled over rain cells or clouds by [1] using an analytical model exhibit similar behavior. A method of identification, detection and localization of these characteristic transient features within the along-track series of these parameters can certainly be used to flag the rain/cloud affected samples.

Matching Pursuit (MP) technique [7] which allows the expansion of a function using a dictionary of elementary functions (atoms), provides a fast and powerful method for decomposing a time-series into a few salient features well localized in time and frequency. It has been quite widely used 
for the identification of transient features in domain such such as electroencephalogram analysis [8], precipitations studies [9], [10] or climatic records analysis [11], [12]. It is thus a good candidate for a fast operational method of rain/cloud flagging.

The method can be defined and tested on the Ka-band simulated waveforms, but it can also easily be tested on real data, i.e. rain affected waveforms measured at $\mathrm{Ku}$-band by Jason-1, which allows a validation against operational dual frequency rain flags [4].

In Section 2, we present the Altika altimeter waveforms modeled in presence of rain and clouds and the typical along track variations of backscatter and off-nadir angle. Section 3 introduces the Matching Pursuit algorithm and the discrete wavelet transform used to generate the dictionary of atoms. It also presents the practical method to flag rain/cloud affected samples. The flag is tested on simulated Ka-band waveforms and on Jason-1 measured Ku-band waveforms in this section. We make some concluding remarks in Section 4.

\section{VARIATION OF ATTENUATION AND OFF-NADIR ANGLE}

Using the model presented in part I of this paper [1], we computed about 20,000 20-Hz Altika waveforms (i.e. one waveform every $580 \mathrm{~m}$ along-track) using several clouds fields as depicted by high resolution $(1 \mathrm{~km})$ cloud liquid water content measurements from MODIS Clouds 5-Min L2 Swath $1 \mathrm{~km}$ and $5 \mathrm{~km}$ Collection 004 and 005 Product [13] (available at http://modis-atmos.gsfc.nasa.gov/MOD06_L2/). A speckle noise of the same order of magnitude as the one measured on Jason-1 waveforms was then added to the Altika simulated ones. For each waveform, the attenuation, $A_{w}$ and the off-nadir angle, $\zeta^{2}$, were then computed. Figure 1 presents an example of Altika waveforms simulated over a MODIS cloud field characterized by a medium liquid water content (maximum of $0.3 \mathrm{~kg} \cdot \mathrm{m}^{-2}$ ) and a large range of cloud sizes. Figure 2 presents the mean and std of the integrated liquid water content (ILWC) over the altimeter footprint ( $\sim 6 \mathrm{~km}$ diameter), the offnadir angle, and the attenuation estimated from the simulated waveforms. The altimeter will of course not measure the signal attenuation but the apparent backscatter $\sigma_{0}$ which is the sum of the true ocean surface backscatter (due to surface wind) and the attenuation caused by cloud. A modeled backscatter (Figure 2-d) was thus computed by adding attenuation and a surface backscatter, here a typical surface backscatter measured by the Jason-1 altimeter. These variations of backscatter and off-nadir angle are similar to the ones observed in $\mathrm{Ku}$ band altimeter data affected by rain such as the one presented in Figure 4 for Jason-1 20-Hz altimeter measurements. The Jason-1 off-nadir angle (Figure 4-b) has been estimated using the same relation as the one used for Altika. Because of the Jason-1 higher altitude and larger antenna beam-width the typical off-nadir angle values are about 4.7 times larger than the Altika ones for the same plateau slope.

The presence of liquid water within the altimeter footprint is associated to coherent and sharp pulses of off-nadir angle and
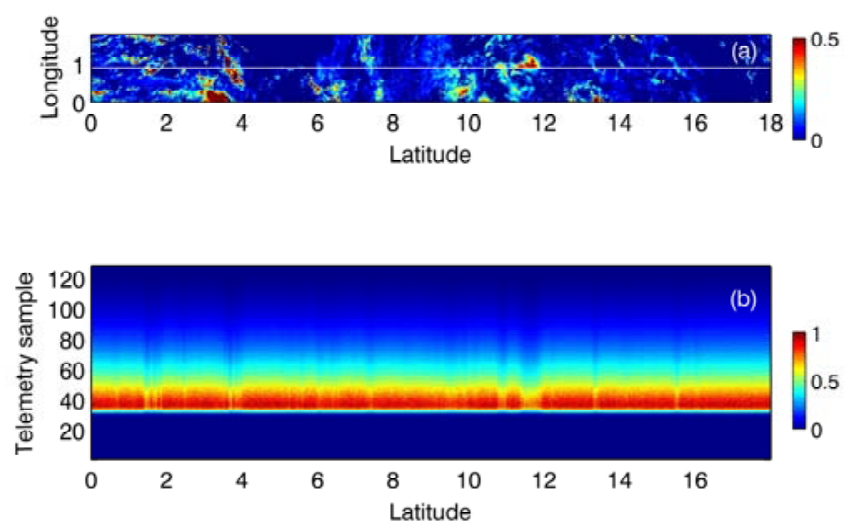

Fig. 1. Integrated cloud liquid water from MODIS/Terra Clouds 5-Min L2 Swath $1 \mathrm{~km}$ data for August 10, 2001 (a). The white line represent the pseudo Altika track. Altika $20 \mathrm{~Hz}$ waveforms simulated along the track presented in (a).

attenuation. Attenuation reflects quite closely ILWC and the characteristics length of the attenuation pulses are of the same order than that of the clouds. The off-nadir angle, which is a good indicator of the waveform distortion depends strongly on ILWC variability within the altimeter footprint and the strongest variations are thus located preferentially near the edges of the clouds. The variation of backscatter is more complex as it reflects not only the variation of attenuation induced by IWLC but also by that of surface wind. If the large scale $\sigma_{0}$ variability is clearly associated to the large scale surface wind variability, the medium and short scale ones result from both liquid water and wind variability. Without some a-priori information, it is thus impossible to separate their relative contributions and the $\sigma_{0}$ analysis can not be used alone for rain/cloud flagging. begincenter The alongtrack off-nadir angle variations are much simpler and they can be decomposed into a low frequency signal associated with the "real" mispointing due to the platform movements (typical length scale of $10000 \mathrm{~km}$ for orbital dynamics or $1000 \mathrm{~km}$ for orbital maneuvers), Gaussian noise associated with speckle and short scale pulse (Dirac). These pulses are associated with waveform distortion caused by strong short scales variations of backscatter within the altimeter footprint created by atmospheric water attenuation. It should be mentioned that as shown by [14], surface slick can also created such pulses but in such cases the signal is not attenuated but strongly enhanced (so that the phenomenon has been called "sigma0bloom" in the literature) because of very high surface backscatter.

The errors induced by clouds on the range and significant wave height estimated by the MLE4 algorithm planned for the waveform retracking [15] are also presented in Figure 2-e and $-f$. The range and swh errors, i.e. the difference between the values estimated by MLE4 and the one used as inputs in the waveform model, result from waveform distortion and the 

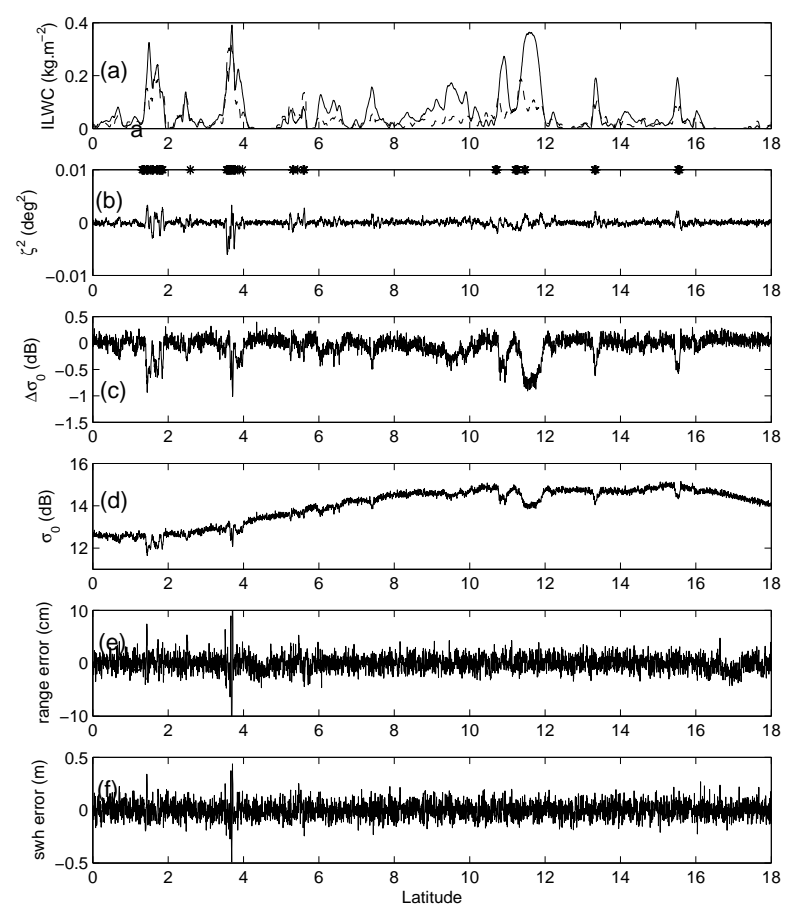

Fig. 2. Integrated cloud liquid water, mean (solid line) and standard deviation (dashed line) over the altimeter footprint (a), Off-nadir angle estimate (b), attenuation (c), backscatter (d), range error (e) and significant wave height error (f) corresponding to the simulated waveforms of Figure 1.
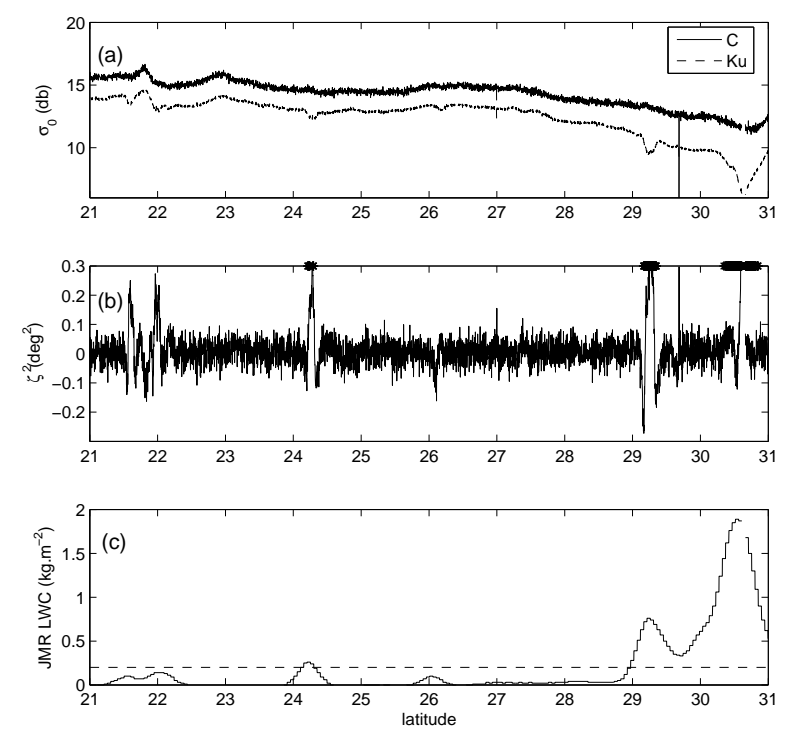

Fig. 3. Example of rain affected Jason SDR measurements (Cycle 62, Pass 152, Sept. 17, 2003). Ku- and C-band 20-Hz backscatter coefficients (a), 20$\mathrm{Hz}$ off-nadir angle estimates, the stars in (b) indicate the samples flagged for rain by the operational Jason rain flag (b) and radiometer liquid water content, the dashed line represents the liquid water content threshold used in the detection algorithm (c).

largest errors are associated to the largest peaks of off-nadir angles as for example near $4^{\circ}$.

Typical attenuation and $\zeta^{2}$ variations induced by clouds have been modeled using simple cylindrical clouds with ILWC from 0.1 to $2 \mathrm{~kg} . \mathrm{m}^{-2}$ and diameters from 2 to $40 \mathrm{~km}$. The relative variations of attenuation and $\zeta^{2}$ (i.e. normalized by the minimum and maximum values) as a function of the distance between the cloud center and the satellite nadir normalized by the cloud diameter are presented in Figure 5. Attenuation presents in all case the same variation, i.e. a sharp drop whose length is close to the cloud diameter. When the cloud diameter is smaller than the altimeter footprint, $\zeta^{2}$ presents a single peaks whilst for larger cloud, the variation is more complex and characterized by two peaks located near the cloud edges where IWLC variability is maximum.

\section{RAIN/CLOUD FLAG}

\section{A. Wavelet analysis and matching pursuit}

Matching pursuit was originally formulated by [7] as a technique for identifying the time/frequency content of a time series whose spectral properties evolve over time. The basic idea was to construct a large 'dictionary' of explanatory functions that are localized both in time and in frequency and then to analyze a time series by projecting it against the functions in the dictionary. Matching pursuit can be adapted to explore other properties of a time series besides its time/frequency content.

Below we briefly recall the basic ideas: we seek a linear expansion approximating the analyzed signal $s(t)$ :

$$
s(t) \approx \sum_{i=1}^{M} a_{i} g_{i}(t)
$$

in terms of functions $g_{i}$ chosen from a large and redundant set of basic functions (dictionary $\mathcal{D}$ ). The problem of choosing $M$ functions, which explain the largest part of variance of a given signal is a $N-P$ hard problem [16], i.e. computationally intractable. MP offers a sub-optimal solution, obtained by means of an iterative algorithm. In the first step, the function $g_{0}$ which gives the largest product with the signal $s$ is chosen from the dictionary $\mathcal{D}$, composed of normalized functions $\left(\left\|g_{n}\right\|=1\right)$. In each of the consecutive steps, the function $g_{n}$ is matched to the signal $R_{s}{ }^{n}$ which is the residual left after subtracting results of previous iterations:

$$
\left\{\begin{array}{l}
R_{s}^{0}=s \\
R_{s}^{n+1}=R_{s}^{n}-<R_{s}^{n}, g_{n}>g_{n} \\
g_{n}=\arg \max _{g_{i \in D}}\left(<R_{s}^{n}, g_{i}>\right)
\end{array}\right.
$$

For a complete dictionary the procedure converges to $s$ with $M \rightarrow \infty$ [7]. In practice we use finite expansions

$$
s=\sum_{n=0}^{M}<R_{s}^{n}, g_{n}>g_{n}
$$

Orthogonality of $R_{s}^{n+1}$ and $g_{n}$ in each step implies the conservation of energy

$$
\|s\|^{2}=\sum_{n=0}^{M-1}\left|<R_{s}^{n}, g_{n}>\right|^{2}+\left\|R_{s}^{M}\right\|^{2}
$$


The complete demonstration of the convergence and energy conservation is given in [7]. The most important feature of this decomposition is that it is a greedy algorithm, i.e. that chooses at each iteration a function that is best adapted to approximate part of the signal.

Many different dictionaries can be used for MP depending on the type of time-series to analyze. As originally proposed by [7] for audio signal, we use wavelets to create the timefrequency dictionary of atoms necessary for MP. The continuous wavelet transform (CWT) of time series $s$ with respect to wavelet $\psi$ is defined as

$$
W(\lambda, t)=\int_{-\infty}^{+\infty} \psi_{\lambda, t}(u) s(u) d u
$$

where

$$
\psi_{\lambda, t}(u)=\frac{1}{\sqrt{\lambda}} \psi\left(\frac{u-t}{\lambda}\right)
$$

The variable $\lambda$ is the scale and $t$ is the point where the wavelet is centered.

For discrete time-series, CWT cannot be computed exactly, and discrete wavelet transform (DWT) has to be used. The DWT can be regarded as an approximation of the CWT over a so-called dyadic grid of scales. Each row is usually set to the largest integer that is less than or equal to $\log _{2}(N)$ where $N$ represents the sample size [17].

Several wavelet analysis and matching pursuit software are available as freeware, among them, Wavelab [18] (available at http://www-stat.stanford.edu/ wavelab/) is certainly one of the most complete and easy to use. The algorithm for rain/cloud detection is defined using Wavelab functions.

\section{B. Rain/cloud detection using Matching Pursuit}

The detection of altimeter samples affected by rain or cloud is based on the analysis of the along-track variations of the off-nadir angle. The goal is here to detect the intervals where the off-nadir angle presents short scales coherent variations such as the ones presented in Figures 2 and 5.

The along track $\zeta^{2}$ resembles a Werner Sorrows signal, i.e. a superposition of sinusoids and Diracs [19] and can be easily decomposed using MP and the dictionary of atoms defined by the wave-packet decomposition of the signal defined by Daubechies 8 (D8) mother wavelet (see Figure 6). This wavelet was chosen because it is quite similar to the typical $\zeta^{2}$ variations presented in Figure 5. The $\zeta^{2}(t)$ series is thus decomposed into a small number of atoms using the Mallat and Zhang MP algorithm, i.e.

$$
\zeta^{2}(t) \simeq \sum_{i=1}^{n} a_{i} g_{i}(t)=\sum_{i=1}^{n} a_{i} \psi_{\lambda_{i}, t_{i}}(t)
$$

where $n$ is the number of selected atoms, $a_{i}$ is the energy of atom $i$ and $g_{i}$ are the atoms chosen from the dictionary $\mathcal{D}$ of the D8 wavelets $\psi_{\lambda, t}$.

In practice, the method can be applied to a time series of any length $m$. As the length is arbitrary, the signal is first padded to dyadic length (i.e. to $2^{m_{1}}$ where $m_{1}=\left\lceil\log _{2}(m)\right\rceil$ ) by
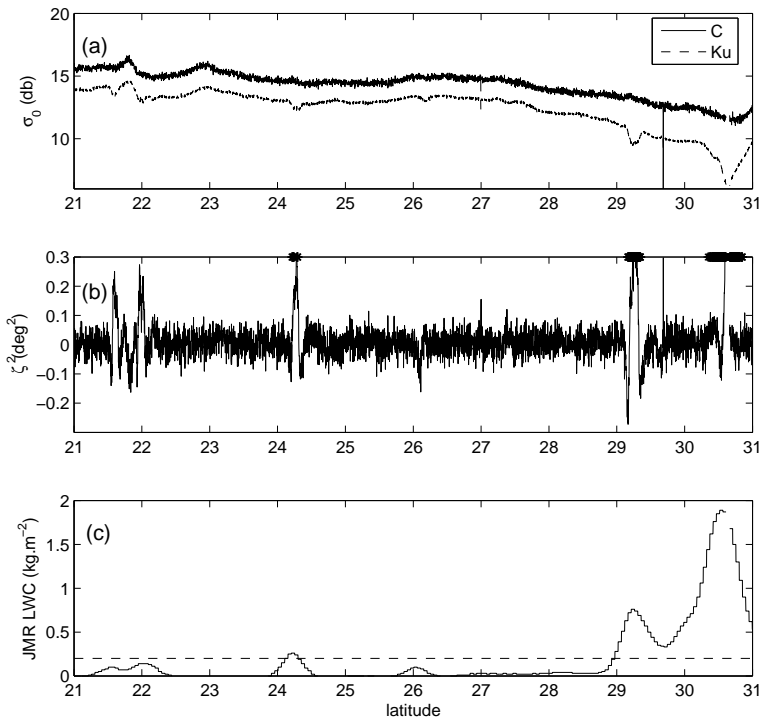

Fig. 4. Example of rain affected Jason SDR measurements (Cycle 62, Pass 152 , Sept. 17, 2003). Ku- and C-band 20-Hz backscatter coefficients (a), 20$\mathrm{Hz}$ off-nadir angle estimates, the stars in (b) indicate the samples flagged for rain by the operational Jason rain flag (b) and radiometer liquid water content, the dashed line represents the liquid water content threshold used in the detection algorithm (c).

folding to allow discrete wavelet transform. The signal is then normalized by the $\zeta^{2}$ noise computed in absence of clouds and rain and it is decomposed over the wavelet packet defined by the D8 mother wavelet. Only the wavelets of scale $\lambda$ less than 9 , i.e. less $\sim 512$ sample (about $160 \mathrm{~km}$ ) length, are considered as longer scales can not be associated to rain or cloud. The MP algorithm is then applied to select the pertinent atoms. The original selection criterion proposed by [7] selects the $N$ atoms which have the largest energy where $N$ is chosen by the user. In absence of cloud or rain such a selection criterion can lead to false alarm as $N$ atoms are always found. It was adapted to select only the atoms whose energy is significantly larger than the noise level.

After testing different threshold values in particular for false alarm rate, the energy threshold was set to three times the noise level. An example of MP decomposition of the along track $\zeta^{2}$ variations of Figure 2 is presented in Figure 7. The twentynine atoms selected are shown in Figure 7-a. Each atom is characterized by its location (Figure 7-a), its energy (Figure 7-d) and its scale (Figure 7-e). Some very energetic events, such as the one near $4^{\circ}$, can be associated to several atoms of different scales and energies, while less energetic ones such as the one near $16^{\circ}$ are well represented by a single atom.

The approximation of the signal by the selected atoms (7) is a filtered version $\overline{\zeta^{2}}$ of the signal that contains only the most energetic short scale variations associated to clouds. Figure 8 which presents $\zeta^{2}$ computed from the noisy waveforms of Figure 1 as well as the ones estimated from the nonnoisy waveforms and the MP filtered ones, shows that the 

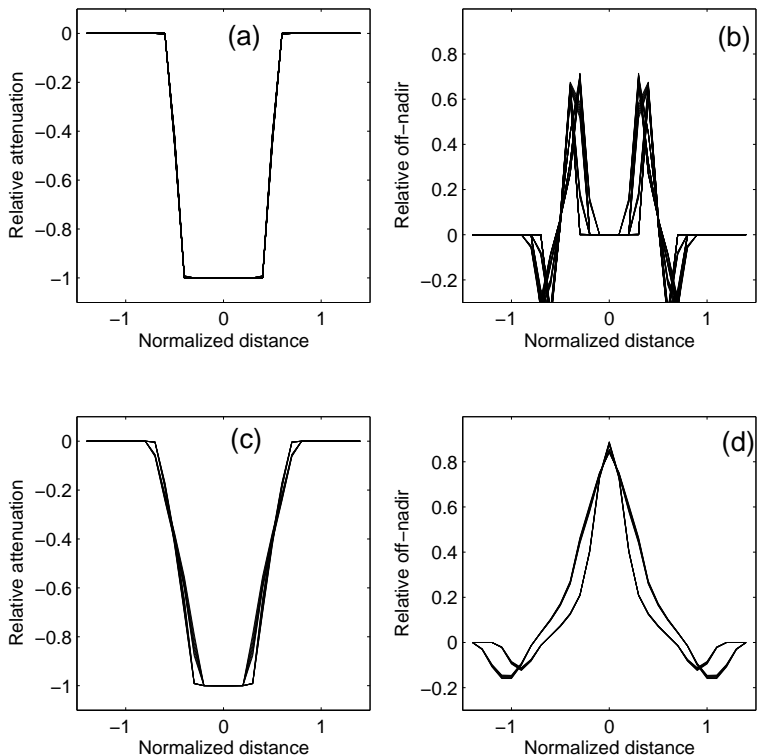

Fig. 5. Variation of attenuation (a) and off-nadir angle (b) as a function of the distance between the satellite nadir and the cloud center for cylindrical constant clouds whose diameters are larger than the altimeter footprint. IWLC ranges from 0.1 to $2.0 \mathrm{~kg} \cdot \mathrm{m}^{-2}$ and diameter from 10 to $30 \mathrm{~km}$. The distance is normalized by the cloud diameter and the variations are normalized by the minimum and maximum values. (c) and (d) idem for clouds with diameter (2 to $6 \mathrm{~km}$ ) smaller than the altimeter footprint.

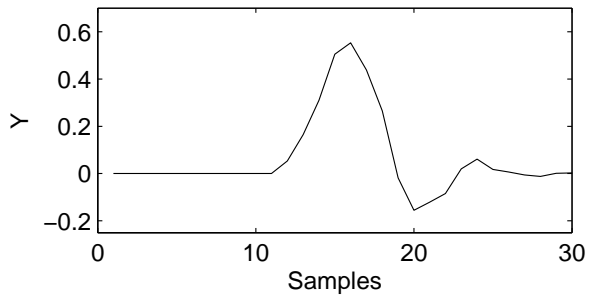

Fig. 6. Daubechies 8 (D8) mother wavelet.

reconstructed $\zeta^{2}$ reproduces very well the variations associated to clouds (i.e. $\zeta^{2}$ estimated from the non-noisy waveforms). The MP $\overline{\zeta^{2}}$ is then used to flag the samples by selecting the values whose absolute value is larger than a given threshold $\left(\left|\overline{\xi^{2}}\right|>\alpha \xi_{\text {noise }}^{2}\right.$ with $\left.\alpha=0.1\right)$. This selection is used to eliminate the small ripples associated located at the edges of some atoms.

The first quality a flag should have is a false alarm rate as small as possible. This has been tested by applying the method to an ensemble of Altika passes simulated in cloud free conditions. More than 20,000 cloud free waveforms composing 6 passes have been analyzed. The MP method detected no atoms and no samples were flagged showing that the false alarm rate is minimum.
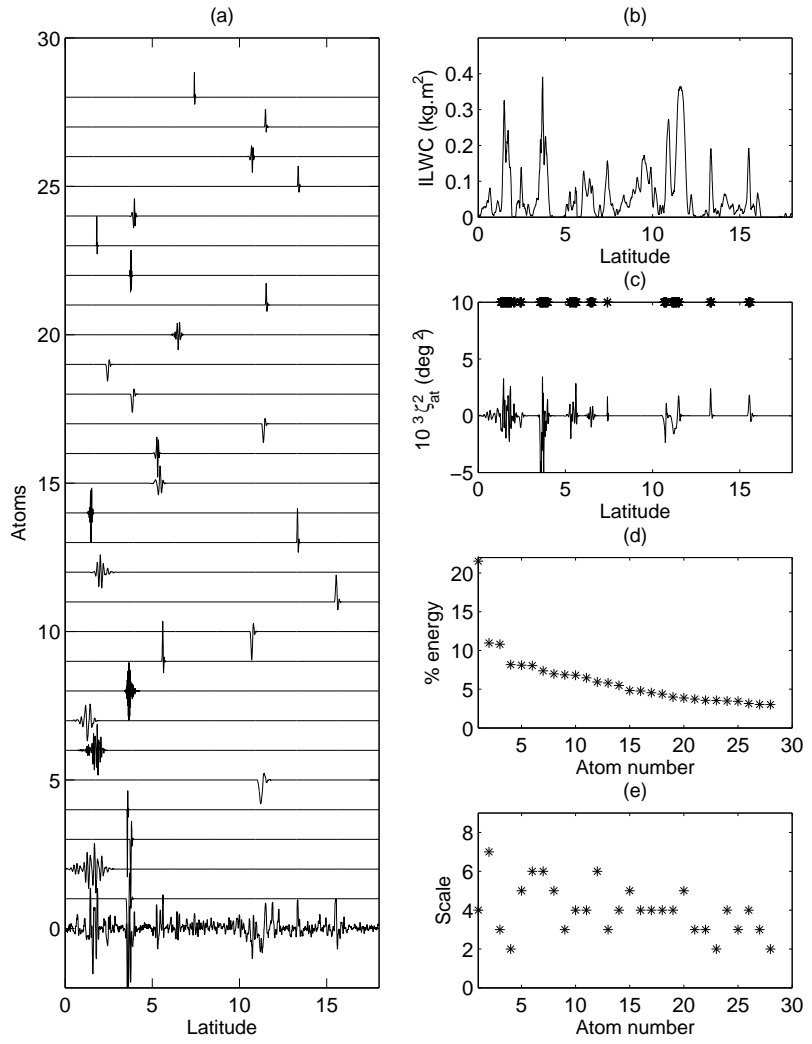

(e)

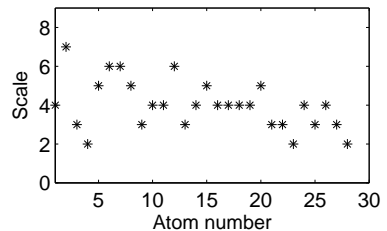

Fig. 7. Matching pursuit decomposition of the off-nadir angle of figure 2 The 29 atoms that pass the energy threshold are presented in (a), the integrated liquid water content in (b), the filtered off-nadir angle and flagged samples (stars) in (c), the energy in (d) and the scale in (e).

The second quality of a flag is its score, i.e. its ability to detect the samples for which cloud/rain causes large geophysical parameters errors (range, swh and attenuation). Errors on geophysical parameters can be decomposed into coherent ones caused by cloud/rain and random ones induced by speckle noise. As we used simulated waveforms, the cloud induced errors are estimated as the differences between the values used in the model and the ones retrieved by MLE4 on the nonnoisy waveforms. The score of the MP flag can be estimated by comparison of the percentage of the flagged noisy and non-noisy samples for given geophysical parameters errors. An ensemble of 15 Altika tracks, totaling more than 60,000 waveforms simulated in cloudy, rainy and cloud/rain free conditions, has been flagged (using the noisy off-nadir angle estimates). For each waveforms, the geophysical parameters errors are estimated by MLE4 for both noisy and non-noisy waveforms. The percentages of flagged samples for given range error, swh error and attenuation are presented in Figure 9. The detection of cloud induced errors is over $90 \%$ for range error larger than $5 \mathrm{~cm}$ and swh error larger than $20 \mathrm{~cm}$, which shows that the score of the algorithm is very good in detecting all the waveforms that are strongly distorted by cloud liquid water. The comparison of noisy and non-noisy percentages of flagged samples, presented in the Figure, shows that the MP 

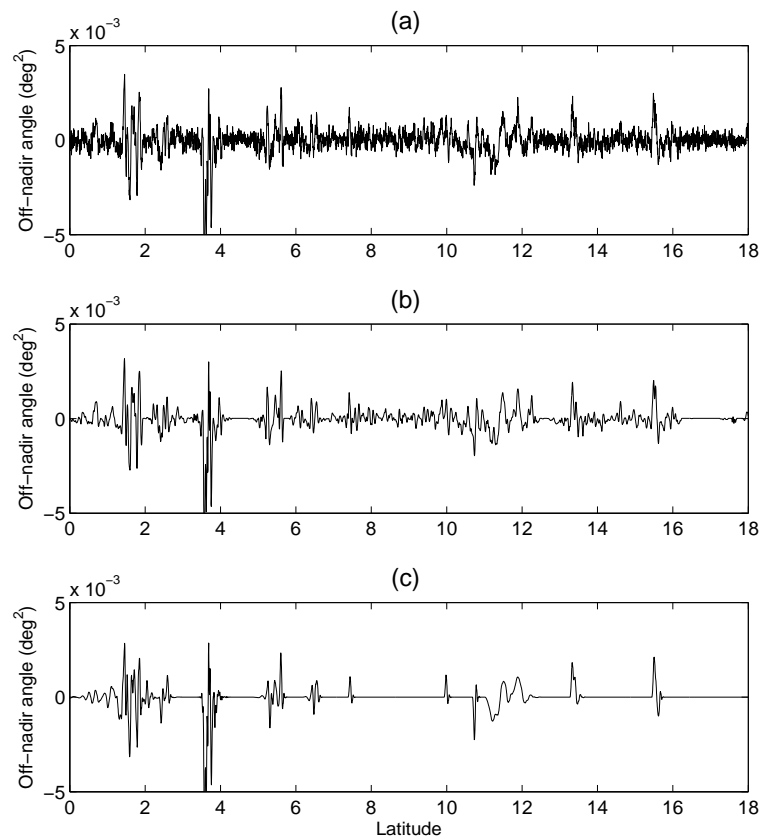

Fig. 8. Off-nadir angle computed from the noisy waveform of Figure 1 (a), from non-noisylyx ones (b), off-nadir angle filtered by the MP detection algorithm (c).

algorithm allows a good discrimination of cloud and noise induced errors. The Figure also shows that no samples with zero IWLC are flagged, i.e. that the probability of false alarm for cloud is null. However, the false alarm rate for range and swh, i.e. percentage of samples having a zero non-noisy range or swh error (or a noisy range error $<2.5 \mathrm{~cm}$ and noisy swh error $<20 \mathrm{~cm}$ ) is about $10 \%$. As the probability of false alarm of non cloudy samples is null, these false alarms for range and swh concern only samples that are located within or near clouds and are only weakly affected.

Concerning attenuation, the MP flag score is good for medium and high attenuation: over $99 \%$ at a $2 \mathrm{~dB}$ level and over $80 \%$ at a $1 \mathrm{~dB}$ level and it is acceptable at low attenuation: about $50 \%$ at a $0.5 \mathrm{~dB}$ level. The percentage of flagged samples for given mean and std of the integrated water content (Figure 9-d) confirms that for non cloudy samples the false alarm rate is null. For high IWLC $\left(>0.5 \mathrm{~mm} \cdot \mathrm{h}^{-1}\right)$ and for high std (>0.25 mm.h $\mathrm{h}^{-1}$ ) more than $90 \%$ of the samples will be flagged which is in good agreement with the results of the analysis of the simulated waveforms presented in part I of the study.

\section{Validation using Jason data}

The method was further tested and validated using real highrate waveform data (SDR) from the Jason-1 altimeter. The MP flag is very versatile and can be very easily adapted to Jason-1 data by simply changing the normalization coefficient of $\zeta^{2}$,
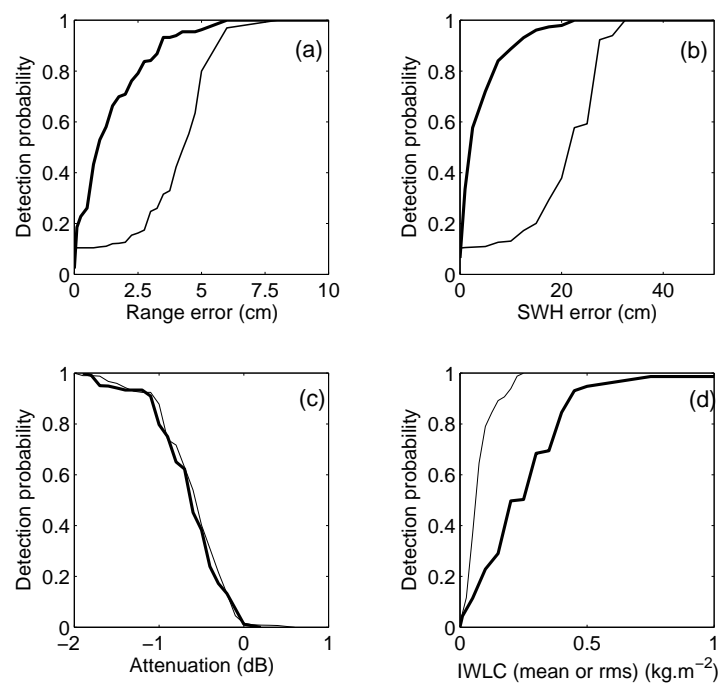

Fig. 9. Cloud/rain flag performances. Percentage of detection for given range errors (a), and swh error (b), attenuation (c). The thick solid line represents the detection for non-noisy waveforms and the thin one for noisy waveforms. Percentage of samples detected for given mean IWLC (thick solid line) and std of IWLC (thin solid line) (d).

i.e. the noise level of the rain-free $\zeta^{2}$. The different selection thresholds are the same as the Altika ones. The results can then easily be compared to the operational flag based on the dualfrequency capabilities of the Jason-1 altimeter. Several passes, totaling 80,000 waveforms have been thus been processed and flagged. The operational Jason-1 rain flag, which is normally given only for $1 \mathrm{~Hz}$ GDR data, has here been applied to the 20 $\mathrm{Hz}$ SDR data for a more precise comparison. A description of this flag is given in Appendix. Figure 10 presents a comparison of the MP and operational flags for the Jason-1 pass presented in Figure 4. The Ku-band attenuation presented in Figure 4-a is the one given by (8) of Appendix. As it is not possible to compute the true range error from measured waveform, we computed a first order approximation of this error in the form of the difference between the $20 \mathrm{~Hz}$ range and the $1 \mathrm{~Hz}$ one (presented in Figure 4-c). All the rain events detected by the operational flag (near $24^{\circ}, 29-31^{\circ}$ ) are detected by the MP flag. The MP flags only the samples strongly distorted and not those that are strongly attenuated and that might still be used to estimate valid geophysical parameters. MP also detects two smaller rain events near $21.5^{\circ}$ and $26^{\circ}$ associated to large waveform distortions and range errors. They were not detected by the operational flag because the liquid water content was below the detection threshold of $0.2 \mathrm{~kg} . \mathrm{m}^{-2}$ (see Figure 4-c), certainly because the rain cells were small. This example shows that the MP flag performs certainly as well as the operational one and that it is certainly more sensitive in detecting small light rain events. It also shows that a sampleto-sample comparison of the two flags, for example in the form of dichotomous discrimination, will not be fully pertinent because the bases of the two flags are too different. The MP flags detects along track structures while the operational ones tests the $\mathrm{Ku}$ and $\mathrm{C}$-band backscatters sample by sample. The 

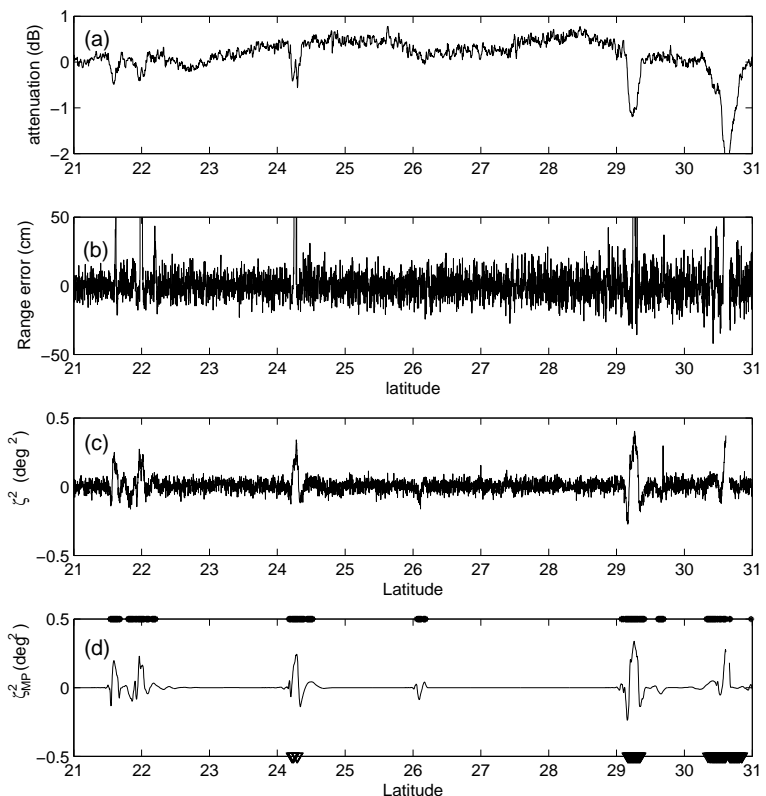

Fig. 10. Rain flag using MP for Jason data. Example of detection for pass 152 of cycle 62 (Sept. 17, 2003). 20-Hz Ku-band attenuation (a) range error (b), off-nadir angle (c) and MP filtered off-nadir angle (d). The upper symbols (crosses) indicate the samples flagged for rain by the MP algorithm and the lower symbols (triangles) the ones flagged by the operational Jason rain flag.

flags' performances are thus estimated by comparison of their respective scores in detecting range errors, off-nadir angles and attenuations. The MP flags has a much better score in detecting high range error as can be seen in Figure 11-a, whilst the false alarm rate is somewhat larger (8\% compared to $4 \%$ ). As it can be expected from the respective flag definitions, the detection of large off-nadir angle by MP (Figure 11-b) is much better than by the operational flag one, while the detection of attenuation (Figure 11-c) is inferior by about $20 \%$. However, MP score is still over $80 \%$ at a $2 \mathrm{~dB}$ level. The percentage of flagged samples for given liquid water content (Figure 11d) is by definition null for low liquid water content (below $0.2 \mathrm{~kg} \cdot \mathrm{m}^{-2}$ for the operational flag and it is about $5-10 \%$ for MP. For higher values, more samples are flagged by the operational flag than by MP but the difference remains limited (about 5\%).

\section{SUMMARY AND CONCLUSION}

The main instrument of the future Altika/SARAL mission will be a wide-band Ka-band altimeter. The major drawback of the use of Ka-band for altimeter mission is its sensitivity to atmospheric cloud liquid water. The analysis of the impact of cloud and rain through analytical model have shown that even for light rain and small cloud the altimeter echo waveforms can be significantly attenuated and distorted leading to erroneous
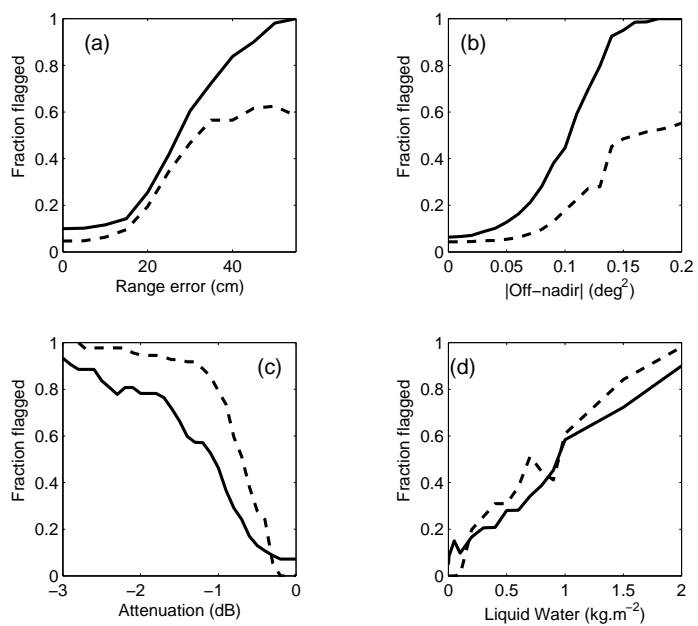

Fig. 11. Comparison of the MP (solid line) and the operational Jason-1 (dashed line) rain flags. Fraction of samples detected as a function of range error (a) absolute value of off-nadir angle (b), attenuation (c) and liquid water content (d).

geophysical parameters estimates. It is thus necessary to accurately detect and flag the samples that are potentially affected by liquid water. Because of the use of a single frequency for Altika, it is not possible to use the kind of rain flag developed and currently used for dual frequency altimeters such as Jason-1. A new rain flag has thus been defined, based on the detection of coherent short scale variations of the offnadir angle estimates (i.e. basically the slope of the plateau of the waveform). Indeed, past studies using Topex and Jason1 data or Altika simulations have shown that rain cells and clouds are always associated with sharp variations of this parameter. The detection algorithm is based on the Matching Pursuit technique which allows the decomposition of a signal into a few salient features using a dictionary of elementary functions, here constituted by the wave-packet decomposition of the signal. The analysis of the algorithm performances using an ensemble of simulated altimeter passes for different cloud conditions shows that MP flag detects more than $90 \%$ of samples with range errors over $5 \mathrm{~cm}$, swh errors over $20 \mathrm{~cm}$ and attenuation over $2 \mathrm{~dB}$. The false alarm rate for cloud is null, i.e. that no samples with zero IWLC are flagged. The false alarm rate for range and swh, i.e. the percentage of sample with range error less than $2.5 \mathrm{~cm}$ and $\mathrm{swh}$ error less than $25 \mathrm{~cm}$ is about $10 \%$. These flagged samples are located near clouds and are only weakly affected.

The MP algorithm is extremely versatile and it can be easily adapted to any altimeter data by simply changing the coefficient used to normalize the off-nadir angle (i.e. the noise level in cloud/rain free conditions). The method has thus been applied to an ensemble of Jason-1 passes. The comparison of the MP and Jason-1 operational rain flag shows that the MP flag performs better in detecting the waveform distortion and thus range errors with a slightly higher false alarm rate, while it performances are inferior in detecting attenuated samples.

The MP algorithm is fast and can easily be coded in the ground processing chain to flag first the high rate waveforms 
and then the 1 second average ones (GDR). During the commissioning phase, the different thresholds used in the atoms selection and the rain/cloud flagging can easily be adjusted as well as the normalization coefficient of the offnadir angle to refine the detection for given range and swh errors.

\section{APPENDIX}

\section{OPERATIONAL JASON RAIN FLAG}

The detection of rain events using dual-frequency altimeter data is well established and is currently used operationally in the Jason processing to flag rain affected altimeter samples. The principle has been described in detail in several studies [3], [6], [4] It is based on the frequency dependency of rain attenuation of the electromagnetic signals. Basically, it detects occurrences where the $\mathrm{Ku}$ band $(13.6 \mathrm{GHz})$ backscatter measurements, $\sigma_{0}$, is significantly attenuated compared to the C-band $(5.3 \mathrm{GHz})$ one. In practice, the measured $\mathrm{Ku}$ band $\sigma_{0}$ is compared to the $\mathrm{Ku}$ band $\sigma_{0}$ that should be expected from the measured C-band $\sigma_{0}$ through a rain free relationship. The rain free $\mathrm{Ku} / \mathrm{C}$ relation, $f$, is determined by binning the $\mathrm{Ku}$ band $\sigma_{0}$ data in intervals of $0.1 \mathrm{~dB}$ of $\mathrm{C}$ band $\sigma_{0}$. The mean, $f\left(\sigma_{0}^{C}\right)$, and standard deviation, $r m s\left(\sigma_{0}^{C}\right)$, are then computed in each bin. The rain events are detected using the following criteria [4]

$$
\Delta \sigma_{0}=f\left(\sigma_{0}^{C}\right)-\sigma_{0}^{K u}>\min \left(0.5 d B, 1.8 * \operatorname{rms}\left(\sigma_{0}^{C}\right)\right)
$$

and

$$
L_{z}>200 \mu m
$$

where $\Delta \sigma_{0}$ is the $\mathrm{Ku}$ band rain attenuation, $\sigma_{0}^{K u}$ is the $\mathrm{Ku}$ band backscatter coefficient, $\sigma_{0}^{C}$ is the C-band backscatter coefficient. The radiometer liquid water content, $L_{z}$, is expressed as a quadratic polynomial of the three JMR brightness temperatures [20]. This second criterion is used to ensure the presence of cloud liquid water and thus to minimize the possibility of false alarm.

\section{REFERENCES}

[1] J. Tournadre, J. Lambin, and N. Steunou, "Cloud and rain effects on ALTIKA/SARAL Ka band radar altimeter, Part I: modeling and mean annual data availability," IEEE Trans. Geosc. Remote Sens., 2008.

[2] J. Tournadre, "Determination of rain cell characteristics from the analysis of Topex altimeter echo waveforms," J. Atmos. Oceanic Technol., vol. 15, no. 2, pp. 387-406, Apr. 1998.

[3] J. Tournadre and J. C. Morland, "The effects of rain on TOPEX/Poseidon altimeter data," IEEE Trans. Geosc. Remote Sens., vol. 35, no. 5, pp. 1117-1135, Sep. 1997.

[4] J. Tournadre, "Validation of Jason and Envisat dual frequency rain flags," Marine Geodesy, vol. 27, pp. 153-170, 2004.

[5] N. Tran, E. Obligis, and F. Ferreira, "Comparison of two Jason-1 altimeter precipitation detection algorithms with rain estimates from the TRMM Microwave Imager," J. Atmos. Ocean. Technol., vol. 22, pp. 782-794, 2005.

[6] G. D. Quartly, T. H. Guymer, and M. A. Srokosz, "The effects of rain on Topex radar altimeter data," J. Atmos. Oceanic. Tech., vol. 13, pp. 1209-1229, 1996.

[7] S. G. Mallat and Z. F. Zhang, "Matching pursuits with time-frequency dictionaries," IEEE Trans. Signal Process., vol. 41, pp. 3397-3415, 1993.

[8] P. Durka, D. Ircha, and K. Blinowska, "Stochastic time-frequency dictionaries for matching pursuit," IEEE Trans. Signal Proc., vol. 49, pp. 507-510, 2001.
[9] P. Kumar, "Role of coherent structures in the stochastic-dynamic variability of precipitation," J. Geophys. Res. (Atmos.), vol. 101(D21), p. 26,393-26,404, 1996

[10] P. Kumar and E. Foufoula-Georgiou, "Wavelet applications in geophysics: A review," Reviews of Geophysics, vol. 35(4), p. 385-412, 1997.

[11] C. Eriksson, A. Omstedt, J. Overland, D. Percival, and H. Mofjeld, "Characterizing the european sub-arctic winter climate since 1500 using ice, temperature, and atmospheric circulation time series," J. Climate, vol. 20, p. 5316-5334, 2007

[12] M. Ghil, M. R. Allan, M. D. Dettinger, K. Ide, D. Kondrashov, M. E. Mann, A. W. Robertson, A. Saunders, Y. Tian, F. Varadi, and P. Yiou, "Advanced spectral methods for climatic time series," Rev. Geophys, vol. 40(1), p. 100, 2002.

[13] W. P. Menzel, R. A. Frey, B. A. Baum, and H. Zhang, "Cloud Top Properties and Cloud Phase - Algorithm Theoretical Basis Document. Products: 06-L2, 08-D3, 08-E3, 08-M3," NASA Goddard Space Flight Center, Tech. Rep. ATBD Reference Number: ATBD-MOD-05, 2006.

[14] J. Tournadre, B. Chapron, N. Reul, and D. C. Vandemark, "A satellite altimeter model for ocean slick detection," J. Geophys. Res., vol. 111, no. C4, p. C04004, Apr. 2006.

[15] L. Amarouche, P. Thibaut, O. Zanife, J.-P. Dumont, P. Vincent, and N. Steunou, "Improving the Jason-1 ground retracking to better account for attitude effects," Marine Geod., vol. 27, pp. 171-197, 2004.

[16] G. Davis, S. Mallat, and M. Avelano, "Greedy adaptative approximations," J. Constr. Approx., vol. 13, pp. 57-98, 1997.

[17] R. R. Coifman, Y. Meyer, and M. V. Wickerhauser, Wavelets and Their Applications. Jones and Bartlett, Boston, 1992, ch. Wavelet analysis and signal processing, pp. 153-178.

[18] J. Buckheit and D. Donoho, Wavelet and Statistics, ser. Lecture Notes in Statistics. Springer-Verlag, New York, 1995, no. 103, ch. WaveLab and reproducible research, pp. 55-81.

[19] A. R. Ferreira da Silva, "Approximations with evolutionary pursuit," Signal Processing, vol. 83, p. 465-481, 2003.

[20] S. Keihm, M. Janssen, and C. C. Ruf, "TOPEX/Poseidon Microwave Radiometer (TMR): III Wet troposphere range correction algorithm and prelaunch error budget," IEEE Trans. Geosci. Remote Sens., vol. 33, pp. 147-161, 1995.

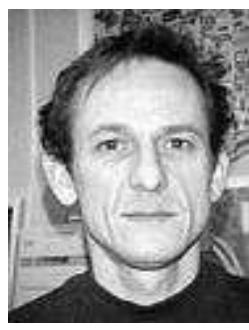

Jean Tournadre was born in Clermont-Ferrand, France, in 1959. He received the diplôme d'ingénieur from the Ecole Centrale de Lyon, Lyon, France in 1981, the Ph.D. degree in Meteorology from the University Blaise Pascal, ClermontFerrand, France in 1984, and the Habilitation á Diriger de Recherches from the Universit $\tilde{A} \odot$ Pierre and Marie Curie, Paris

$\mathrm{He}$ has been post-doctoral researcher at the Scripps Institution of Oceanography, University of California, San Diego (1984-1986) and visiting scientist at the National Center for Atmospheric Research, Boulder (1987), at the University of Hawaii (1997), and at the University of Tohoku, Sendai, Japan (2003). Since 1987, he is a research scientist with the Institut Français de Recherche pour l'Exploitation de la Mer (French Research Institute for Exploitation of the Sea), Plouzané, France, where he works in the Laboratoire d'Océanographie Spatiale.

His research interests are sea surface temperature, microwave remote sensing of the ocean, especially surface winds and waves, and the interaction between radar signal and precipitation.

Juliette Lambin-Artru received the engineer degree form Ecole Polytechnique, Palaiseau, France in 1997 and the Ph.D. in Geophysics from Institut de Physique du Globe de Paris, Paris, France in 2001.

From 2001 to 2005 she was a Postdoctoral Fellow at the California Institute of Technology, Pasadena, CA. In 2005, she joined the Centre National d'Etudes Spatiales in Toulouse, France, as a mission engineer for oceanography, hydrology and cryosphere. She supervised 
Nathalie Steunou was born in France in 1974. She received an Engineer degree from Ecole Nationale de l'Aviation Civile, Toulouse, France in 1996.

In 1996 she joined the Centre National D'Etudes Spatiales (CNES) in Toulouse, France where she works as a radar engineer for altimeters and radiometers oceanographic satellite missions. . 